\title{
Structural Certification of Human-Rated Inflatable Space
} Structures

\author{
Thomas C. Jones \\ NASA Langley Research Center, \\ Hampton, VA 23681
}

\begin{abstract}
This paper details the results of an initial study to develop a certification plan for human-rated inflatable space structures, including guidelines for qualification testing. Habitable softgoods inflatables are multi-layered shell structures that use high-strength webbing, cordage and broadcloth fabric to carry the skin loads of a variety of volumetric shapes and structural architectures. The primary objectives of this study are to define the key parameters that affect these structures and propose a statistically robust approach to defining safety and knockdown factors based on test and analysis. Current NASA standards for habitable inflatable space structures use a factor of safety of 4 , which was inherited from airship design criteria. An updated approach to defining a design factor, taking into account material strength variability, load variability in the article, number of test samples, and damage and degradation effects is specified. Accurate analytical modeling of these structures is hindered by the difficulty of obtaining accurate and consistent material data due to loadhistory-dependent, nonlinear load versus strain behavior. A building block approach to certification is detailed that uses stochastic modeling and statistical test design and analysis to address the unique challenges these high-strength softgoods structures present. Human-rated inflatable modules are a transformative capability for launching much larger habitable volumes into space than is possible with rigid shell structures. This research aims to provide the framework for certifying these structures for future human space exploration missions.
\end{abstract}

\section{Introduction}

Habitable inflatable space structures have been designed and tested for over five decades for application to, and enhancement of, human space exploration. These structures include space stations, habitats, airlocks and deployable tunnels for missions, both in space and on planetary surfaces. They provide the primary benefits of a high packaged-to-deployed volume ratio and reduced mass, based on the use of high specific strength materials and the ability to design them for their deployed pressurized state versus typical launch load constraints. This increase in packaging efficiency and reduction in mass enables the use of a wide variety of launch vehicles and enables a broad range of mission concepts for human space exploration. Current standards provide little guidance on certifying these types of inflatable structures other than defining a factor of safety (FOS) of four on the design pressure, which was inherited from airship design criteria. Given the criticality and desired long-term use of these human-rated inflatable pressure vessels, and the complex, nonlinear behavior and interactions of the high strength softgoods employed in their design, a more detailed and robust approach to certification is needed. An updated approach to defining a design factor for these structures is proposed that takes into account material strength and load variability, number and type of tests, and effects of damage and degradation over time. This paper provides a background on the development and use of human-rated inflatable structures, and the behavior of high-strength softgoods. A review of current standards and certification approaches is then presented with an overview of applicable design, safety and 
knockdown factors. Finally, a statistically robust approach for determining the overall design factor for these structures is presented based on efficient test design and stochastic modeling and analysis.

\section{Research and Testing of Human-Rated Inflatable Space Structures}

Concepts for habitable inflatable space structures are older than NASA itself, going back to Wernher von Braun's rotating wheel space station ${ }^{1}$ in 1952. This $250-\mathrm{ft}$ diameter, three level, rotating toroid, consisted of 20 inflatable sections that would be connected and deployed on-orbit (Figure 1). The primary motivator for using inflatables then still holds today: to provide a large deployed habitable volume that could be compactly packaged for launch. Shortly after NASA's establishment in 1958, Langley Research Center (LaRC), in partnership with Goodyear Aerospace, began investigating inflatable toroidal space stations (Figure 2). Throughout the 1960's, Goodyear performed the first in-depth study of expandable inflatable space structures intended for human spaceflight $^{2,3}$ producing several full-scale test articles that included two lunar surface habitat concepts (Figure 3) and an airlock, in addition to its toroidal space station demonstrators. This program was one of the first to address the challenges of designing and fabricating a multi-layered, human-rated softgoods inflatable that was robust against micrometeoroid and orbital debris, radiation and the thermal environment of space. Sadly none of these concepts were taken to flight. During this same period, the Russians also manufactured an inflatable airlock, called Volga, and launched it in 1965 on Voskhod-2 to enable the first spacewalk in history. Although successful, several life-threatening events during the mission, and a move toward depressurizing the capsule for future extravehicular activity, led to Volga being abandoned. It remained the only human-rated inflatable to go to space until 2016.

The next major human-rated inflatable research program didn't occur until 1997 with the instigation of the Transit Habitat (TransHab) program at NASA's Johnson Space Center (JSC). TransHab was a 3-level, $36 \mathrm{ft}$ long by $27 \mathrm{ft}$ diameter inflatable habitat designed as an element of a larger transit vehicle for Mars, but it was also proposed as a node for the International Space Station (ISS). Between 1997 and 1999, multiple full-diameter restraint layer articles were hydrostatically tested, a full-scale article was packaged and deployed in vacuum and significant research into the design and fabrication of all layers of the inflatable shell (Figure 4) was performed ${ }^{4-6}$. The bladder materials were studied by the International Latex Company (ILC) Dover ${ }^{7}$, who had considerable experience with softgoods and thin films from fabricating the Apollo space suits for NASA. ILC created a low permeability bladder that was robust to packaging and extreme cold. The Micrometeoroids and Orbital Debris (MMOD) layer was extensively analyzed and tested ${ }^{8-9}$ by NASA JSC to provide a soft, compactable Whipple shield that proved to be more effective than the MMOD shield on the ISS. Multi-layer insulation (MLI), and internal scuff and spacer layers were also studied to provide the necessary protection, packageability and positioning required of the inflatable shell. The primary structural restraint layer consisted of a plain weave of highstrength Kevlar webbings, so arranged to reduce the amount of stitching required. Basic strength and stiffness data was obtained, but an in-depth study of the nonlinear, load-history dependent material behavior and the effects of damage and long term degradation to the restraint layer was 
not conducted during the TransHab program. The patents from TransHab were licensed by Bigelow Aerospace (BA) in 2000 and, after significant investment by BA into developing the technology, NASA and BA successfully launched, attached and deployed the Bigelow Expandable Activity Module (BEAM) on the ISS in April 2016.

Research on high-strength inflatable modules at NASA since 2000 has focused primarily on the design, test and analysis of the restraint layer. There are many possible geometries and architectures of the restraint layer used in these structures that combine webbing, cordage and broadcloth fabric. The four most common configurations that have been studied by NASA and its industry partners are shown in Figure 5. The close basket weave approach was used on TransHab and subsequent test articles at NASA JSC. Webbings are woven into a tight mat that is highly robust to damage and allows the use of a non-structural bladder layer. Two separate tests performed on the article shown in Figure 5.a, cut one and four adjacent webbings (two in each principle direction) respectively, via linear shape charges. The result in both cases was negligible movement of the surrounding webbings and no detectable change in the loads measured at the bulkheads ${ }^{10}$, due to the high friction between webbings. The disadvantage is that the load distribution is highly non-deterministic. The second approach is non-woven, with the hoop webbings stitched together along their adjacent edges, and axial webbings overlaid. This simplifies the fabrication process but can lead to complex local stresses in the stitched webbings that again makes load determination challenging and seam design especially critical. The third approach uses an open webbing mesh with an underlying structural fabric. This is typically a lighter construction but it loses some of the robustness of the first two approaches and is again subject to indeterminate loads due to the indexed cross-overs and interaction between the hoop and axial webbings. The last approach uses a lowhoop stress geometry similar to circular army parachutes and super pressure balloons, whereby the principle loads are unidirectional. The low hoop loads are carried by lobes formed between the cordage. This is one of the lightest approaches possible and the principle load paths are determinate, but they are also not redundant by definition. Therefore this design relies on a properly offloaded and seamed fabric layer designed to take the dynamic load of a cord failure, and the cords must be sized to carry the additional load from a failure to add robustness. All restraint layer designs have pros and cons based on mass, cost, ease of manufacture, robustness, load determinacy, and ability to model and verify the structure. Any certification approach has to be able to account for and be customizable to the variety of architectural approaches possible.

\section{High-strength Softgoods Properties and Challenges}

The restraint layer is the most critical, and least well characterized layer of these inflatable modules due to the complexity of the behavior of high-strength softgoods products like webbings, cordage and fabric. These products are hierarchical structural architectures unto themselves, made up of high-strength polymer fibers and yarns. An inflatable article is an even higher level configuration of those softgoods products. Figure 6 illustrates the five levels of structural hierarchy present in a typical human-rated inflatable module. Influence factors that affect the softgoods structure and properties are present at each level, such as manufacturing processes and sizings used at the fiber and yarn level, weave type and resin selection at the webbing level and weave, friction, 
and stitch properties at the inflatable module level. The parameters across all levels combine to produce the nonlinear mechanical behavior of the components and full-scale article.

The typical load versus strain behavior of a high-strength softgoods product is shown in Figure 7. When the pristine material is first loaded (a) it displays its softest, nonlinear stiffness curve due to decrimping or untwisting of the relatively loose fibers and yarns until fiber-lock is reached (around $40 \sim 50 \%$ of the ultimate load). Above this load the behavior transitions from constructional strain dominated to being primarily elastic strain of the yarns and the curve is more linear. If the specimen is then load cycled a number of times (preconditioned), the curve becomes stiffer (b) and more consistent as the fibers stay partially locked together (even after unloading) and the stress distribution in the fibers becomes more uniform. If the specimen is left unloaded for a period of time, to emulate the time between manufacture and final deployment of the built-up article, the curve shifts to the right (c) due to partial relaxation or recovery of the crimp or twist. This makes the conversion of strain to load, to track load distribution, extremely challenging for space inflatables as often the preconditioned curve (b) is used in modeling and calibration. Methods for accounting for this variation in stiffness behavior for modeling purposes are discussed in section 6 using stochastic analysis.

The restraint layer is ordinarily constructed from commercial off-the-shelf (COTS) softgoods built to military or industry standard specifications ${ }^{11}$. These specifications define the number, denier and construction architecture (layup) of the fibers and yarns that produce a minimum ultimate tensile strength (UTS) with high confidence. The entire lot is discarded if any single specimen breaks below the minimum specified UTS from the same manufacturing run. The most common industry specification used ${ }^{11}$ was created for Kevlar, thus using a different material, even if nominally similar in strength and stiffness can also effect performance. For high-strength webbing and cordage, with high fiber / yarn counts, there can be large variances in the tested UTS around an average value, and that average can be 15 20\% higher than the minimum specification. The stiffness behavior is also affected by this inherent variance, particularly if only pristine (uncycled) material is tested. At NASA LaRC a large number of Vectran webbings ( 90 rolls) have been tested with the following results: 6,000 lbf-rated (6K) webbing had an average 5.9\% strength variation about the mean with a maximum of $11.3 \%$, while 12,500 lbf-rated (12.5K) webbing had an average $9.3 \%$ strength variation about the mean with a maximum of $21 \%$. A large variation in strength reduces the structural efficiency of an article by requiring a designer to use a larger margin on the design load due to the higher uncertainty; this has a significant impact on the predicted creep time to failure (TTF).

The long term behavior of the restraint layer was one of the highest priority areas of required research identified at the end of the TransHab program. Polymeric softgoods used in these structures demonstrate viscoelastic creep behavior (Figure 8) that must be characterized for each inflatable design, especially for those proposed for use on long duration missions. Real-time creep testing of high-strength softgoods is costly and requires a large, environmentally controlled test facility that can house a large number of specimens for multiple years. Due to the high load capacity of the webbings and cordage used in these structures, large weights are typically required to perform the tests. Creep tests are run at different percentages of the UTS for each type of softgoods used in the article to produce a plot like the one shown in Figure 9 for the $6 \mathrm{~K}$ and $12.5 \mathrm{~K}$ 
Vectran tested at LaRC. Typically five or more specimens are tested at each \% UTS to ensure an accurate bracketing of the strength values; this is crucial if high variance is observed. As noted, the $12.5 \mathrm{~K}$ webbings have higher strength variance than the $6 \mathrm{~K}$ webbings, which is reflected in the wider band of TTF for the $12.5 \mathrm{~K}$. This wide range of TTF is due to the load at a chosen \% UTS being based on the tested average, so an individual specimen may have a higher or lower actual UTS within the tested variance for that roll. As TTF is related exponentially to the load level, even a small percentage change in the load can lead to a dramatic change in the TTF.

Non-uniform load distribution in the restraint layer can also have a strong impact on TTF. As an example, if one wanted to look at TTF at $80 \%$ UTS for $12.5 \mathrm{~K}$ Vectran, the range of TTF is from 12 minutes to 250 hours (10.4 days). If we assume a relatively minor $\pm 5 \%$ load variation in the webbings such that the loads are actually 75\% to 85\% UTS then the TTF range broadens to 4 minutes up to 5.5 months. 80\% UTS was chosen for convenience, but actual inflatables would currently operate at $25 \%$ UTS, where there is a significantly wider spread in TTF predicted. NASA LaRC is currently preparing a report on the most efficient approaches to performing real-time creep testing on high-strength softgoods specimens based on lessons learned from a 5-year creep test program that assessed multiple setups and approaches. Accelerated creep testing methodology for high strength softgoods was also pursued during the creep program, to reduce the cost and time required to produce vital creep data and is being resumed this year at NASA LaRC. Creep is one of the most significant factors that could drive the design of future long-duration human-rated inflatables, thus continued research on both accelerated and real time creep methodology is critical.

\section{Review of Relevant Structural Certification and Standards Documents}

The FOS of four used for human-rated softgoods structures was originally appropriated from the only other human-rated inflatable design criteria available; that of fabric airships. The FAA-P-8110-2 airship design criteria ${ }^{12}$ defines an ultimate strength requirement on the pressurized envelope of "not less than four times the limit load determined by the maximum design internal pressure combined with the maximum load resulting from any of the requirements specified" which includes flight and ground conditions, and aerodynamic pressures. This is for pristine, serviceable fabric envelopes that are at a significantly lower stress than the high-strength softgoods architectures used in human-rated inflatables. NASA's primary structural design criteria document, NASA-STD-5001B - structural design and test factors of safety for spaceflight hardware $^{13}$, defines the same FOS of four on habitable softgoods structures (where loss of life is possible) and also specifies it as a minimum "unless adequate engineering risk assessment is provided that justifies the use of lower values". A service life factor of four is also specified on creep and fatigue but, "for materials that are not well characterized, or those [with] complex failure modes, an additional factor and testing may be required.” NASA JSC has their own version of this document, JSC-65828B ${ }^{14}$ that further specifies that the design limit load be multiplied by 1.15

prior to reading the creep curve to account for material data scatter, thus essentially defining a knockdown factor as a required component in addition to the service life factor of 4 . Note that this is a non-material specific requirement, thus the higher variance inherent in high-strength softgoods may require a review of this specification. 
JSC-65828B has more in-depth definitions and requirements than NASA-STD-5001B in several areas beyond the service life factor that are relevant here. One area is in dealing with combined loads, where the analyst is required to determine if any combined loading effect is additive or relieving. This could be difficult to assess for softgoods structures without testing, especially for parameters such as friction or thermal-mechanical, and this is addressed in Section 6 . In addition, JSC-65828B requires math models to be within $10 \%$ of tested values for deflections, stresses and strains. This has been challenging to accomplish to date, partly due to the complexities and non-deterministic nature of these architectures but predominantly from the difficulty of obtaining or quantifying accurate input data on the component materials and interaction behavior between elements of the restraint layer. As detailed in Section 3, the material behavior is nonlinear, load-history and time dependent. Frictional and seam effects are hard to quantify a priori, and even more challenging to measure directly on the as-built article. Fabrication tolerances, which are orders of magnitude higher than rigid structures, create another load path variance. NASA's standards were developed for structures with materials that are well-characterized for their service environment and design conditions. Analysis and test correlation for high-strength softgoods structures will require novel, and most likely stochastic or probabilistic approaches to bound the tested behavior.

NASA has an extensive set of additional standards for rigid space structures, particularly for defining requirements on human-rated structures where the criticality of failure is high. SSP30559 and SSP-30558 define the structural design and verification ${ }^{15}$, and fracture control $^{16}$ requirements for all space station hardware and payloads. Although not specific to softgoods structures, they do define relevant attributes that would be required of any long duration humanrated inflatable habitat. These include defining fail-safe behaviour, or safe-life, that specifies a service life factor of four on the required 15 year service life on station. Fail-safe for an inflatable structure could be classified as leak before break, or the ability to redistribute load after a single primary structural member failure, such as a cord or webbing. A 15 year service life requirement for human-rated inflatables is a realistic possibility for proposed future long duration applications such as planetary outpost elements or large in-space habitats and stations. SSP-30559 also specifies that periodic maintenance, inspection and repair could be used to address this length of mission, but that may be impossible for multi-layered inflatable structures unless the layers are designed to be modular or serviceable.

Many of NASA's standards, including SSP-30559, SSP-30558, and JSC-65828B, describe using $\mathrm{A}$ and B-basis allowables for material acceptance. Commonly used for metals and composites, these statistically-based material properties rely on a significant test database on the material in question, which typically doesn't exist for high-strength softgoods. An A-basis allowable represents a parameter value that $99 \%$ of the population will meet or exceed with $95 \%$ confidence (often applied to single point failure). A B-basis allowable represents a parameter value that $90 \%$ of the population will meet or exceed with 95\% confidence (applied to redundant structure). An S-basis represents a minimum specification for acceptance but isn't based on a statistical approach. The S-basis is closest to what is currently used for COTS softgoods, on strength and width. No statistical minimum or allowable is set on any other mechanical property such as stiffness. This is likely due to the lack of necessity for high precision property values for 
most applications and the relatively small amount of product manufactured and tested in any one specification. Given the variety and variability currently in high-strength softgoods products, the lack of statistically validated material performance is a big driver in increasing uncertainty in the final article's behavior. Collaboration and buy-in from the manufacturers would be needed to reduce the inherent variability seen today based on minimum strength specifications. Human-rated space inflatables are one of the few applications of high-strength softgoods that would truly benefit from tighter allowables, but the cost and time required of the fabricators to improve the performance of these softgoods products is unlikely to be offset by the quantity required by NASA and its industry partners who make these structures currently.

As only the second habitable inflatable structure ever to be launched and operated, the BEAM article that launched to the ISS in April 2016 lacked the availability of a standard for certification. Instead, BEAM was certified for flight based on a custom ISS interface control document (ICD) ${ }^{17}$ prepared internally at NASA and a structural verification plan and fracture control plan (both proprietary) prepared by Bigelow Aerospace and signed off on by NASA technical authorities overseeing the program. The ICD follows previous requirements documents on interfacing modules with the ISS in a safe manner. The structural verification and fracture control plans use requirements from the standards discussed above including, NASA-STD-5001, SSP-30559 and SSP-30558, referencing the relevant sections in those documents to the BEAM design. These incorporate the required FOS of four on strength, and a safe-life of four times the expected 2-year mission length. The end goal of the current study is to develop a standard certification approach that can provide more detailed requirements than currently specified in the cited standards that are specific to the influence factors of human-rated inflatables structures so future modules have a dedicated standard to follow.

Finally, two pertinent certification approaches for inflatables were found as part of this review. The first was a NASA proposal on certifying expandable surface habitats ${ }^{\mathbf{1 8}}$. This document approaches certification in the same manner as the FAA for composite aircraft, specifying a FOS that was not implemented as a catch-all for all uncertainty and influence factors. Rather, the endof-life (EOL) material properties are determined, based on expected damage and degradation. These EOL properties then have a FOS of 2 added that is in line with NASA requirements on critical pressure structures and parachutes. This incentivizes the designer to both better understand their design approach, and the impact of various factors that could be mitigated, reducing the penalty of EOL knockdowns over time. The second document is a white paper written by the NASA Engineering and Safety Center (NESC) on developing design factors of safety and a technical standard for the Hypersonic Inflatable Aerodynamic Decelerator (HIAD) ${ }^{\mathbf{1 9}}$. HIAD shares a lot of the same material and structural complexities of human-rated inflatable modules, but is a one-time use, extreme temperature application. The NESC team suggested a design factor approach for HIAD that defines a FOS with additional derating factors based on a risk and impact evaluation of possible damage and degradation. These factors would be quantified via structural testing of the components and full-scale system. Both of these papers suggest a similar approach to separating the safety and knockdown factors and testing the identified factors for a given design to determine impact. A design factor approach is also implemented in the current study. 


\section{Design, Safety and Knockdown Factors for Human-Rated Inflatable Structures}

A structural design factor is a combination of a FOS and any additional and relevant derating or knockdown factors based on the damage and degradation expected over the duration of the structure's life, from fabrication through the end of mission. Currently the FOS of four on habitable softgoods structures, as specified in NASA-STD-5001B, takes the place of a generalized design factor by being universally applied to any human-rated inflatable architecture. It assumes a high degree of uncertainty and criticality, but it doesn't account for specific features of a design, mission type, operational environment or prior experience that might necessitate an increase or decrease in the overall factor. In contrast, a design factor can be customized to a particular architecture and mission, and can be systematically reduced over time as experience is gained and best practices are developed and implemented at all stages of fabrication, testing and analysis.

\subsection{Factor of Safety}

The FOS specifies the actual failure load versus the intended design load of the structure and is set based on the level of criticality of the structure, its application, and the relative risk assessed from the level of testing and analysis that has been performed on similar structural architectures and materials. Human-rated inflatables are at the nexus of several high criticality risk areas; they are high-strength, nonlinear softgoods pressure vessels designed for long duration human habitation in an extreme environment (in space or on a planetary surface). Human-rated structures are classified as critical due to possible loss of crew (LOC). These characteristics of habitable inflatables require a higher baseline FOS than metallics and composites, prior to any additional knockdown factors. With appropriate investigation and characterization of the derating factors below however, a baseline FOS of 2 to 3 should be feasible.

\subsection{Knockdown / Derating Factors}

Knockdown factors are all architecture and material dependent at both the component and system level. It is therefore not possible to create a universal design factor for these structures. Each design has to be evaluated against possible influence factors and those identified as relevant must be included in the test and analysis program for that design. Material selection should be based on resistance to the loss factor properties listed in this section. The primary elements that are used to determine loss and load factors that contribute to a combined knockdown factor are listed below along with several additional risk factors that can influence the final design factor for an inflatable article.

5.2.1. Loss factors (LsF) - These are damage and degradation factors that reduce the effective strength or lifetime of the inflatable article.

\section{a) Thermal-vacuum effects}

- Temperature effects on strength from both cold and hot environments in vacuum. Primarily during the launch, transit and initial deployment from a packaged state. Once inflated the restraint layer should see a relatively benign and stable thermal environment due to its position in the shell layup near the interior. Airlock applications require more extensive testing to characterize the knockdowns for repeated thermal exposure and cycling over the 
life of the article. Embrittlement and changes in inter-fiber frictional properties can occur as coatings degrade due to off-gassing in vacuum. Materials and coatings are chosen to minimize these effects.

\section{b) Ultraviolet (UV) light exposure}

- Some materials used for high-strength softgoods are susceptible to degradation from UV exposure. As the restraint layer is sandwiched between multiple layers in the inflatable shell, the primary UV risk is during fabrication and check out. This factor can be minimized by keeping exposed components covered when possible, checking UV output from lights in the fabrication facility and making sure components are not exposed to direct sunlight.

\section{c) Radiation}

- Polymeric softgoods can be degraded by radiation over time. Exposure level and impact is dependent on mission location and length. Radiation is difficult to mitigate without adding significant mass, hence radiation protection for the crew is likely to be restricted to interior storm shelters around the sleep quarters. This will exclude the restraint layer from radiation protection thus radiation effects must be characterized for the expected mission dosage and intensity.

\section{d) Folding from packaging}

- Folding or creasing softgoods materials while stored or packaged. This can damage fibers in the softgoods and lead to premature failure at the crimp points. Several variables including fold radius, pressure on fold, time folded and temperature can impact the severity of the issue. Mitigation could include analytically determining fold lines for lowest stress, increasing fold radius, or investigation of other novel packaging geometries.

\section{e) Abrasion}

- From relative motion between elements in the restraint layer and the layers above and below it during packaging and deployment, particularly from integrated hard structure like hatches or windows, or induced via an internal or external agent (human or robotic) interacting with the structure. Abrasion can be minimized by material or coating selection for lower friction, precise integration, packaging and deployment scheme with offloaded and secured hard structure, particularly for launch.

\section{f) Tear strength}

- Due to cut or puncture of a structural softgoods member, including fabric, webbing or cordage. Primary sources could be impact particulates due to an MMOD layer failure (this would not be a primary requirement on the restraint layer), or stitched joint failure and tear propagation along a seam. Tearing is possible in webbing and cordage, but due to these products being $90+\%$ axial fibers a cut tends to immediately reduce the strength of the product by approximately the percentage of axial fibers lost, with limited propagating 
tearing occurring up to failure. For fabrics, tearing is minimized by using an uncoated fabric or a low adhesion coating on the fabric, integrating ripstops, or using an architectural design that doesn't require a fabric layer.

\section{g) Joining efficiency}

- Seams, stitches, and splices at terminations and connection points between material components can reduce the structural efficiency of the softgoods members. Joints also include RF welded, heat sealed or adhered fabric joints (if coated). Joining softgoods to hard structure, such as hatches or windows, requires a lot of stitched or spliced webbing or cordage terminations to transfer load into the hard structure. Additionally integrating sensors via bonding, additional fibers or wires for sensing in the layup, and inline sensors like load cells for testing can also reduce efficiency in the structural load paths.

- Mitigation is primarily based on experience working with the materials and determination of an efficient stitch pattern for a particular softgoods product and architecture. Generally a zig-zag or diamond pattern has been used in the past, with a lap or fell seam for webbing and fabric. High seam efficiency factors include aligning the stitches in the direction of loading, and using the same material for the thread as the softgoods being joined to eliminate problems caused by different abrasive, frictional or stiffness properties that can lead to premature stitch or fiber failures.

\section{h) Fatigue}

- Cycling of loads on the restraint layer from repeated pressurizations / depressurizations over time. This would primarily be a factor for an airlock or temporary inflatable annex on a manned mobility element like a rover. For these types of applications, fatigue testing would likely replace the creep testing requirement to account for long term material degradation and maintain safe-life requirements. It's believed that cycling the pressure and loads in the restraint layer occasionally with long periods of lower operational pressure (standby, unmanned) is likely less detrimental than creep over a mission life. Issues from snagging, or incidental damage to the layers while unpressurized may be of concern also.

- Large mass(es) with low frequency, such as large hatches, connected solar arrays, or another spacecraft getting dynamically excited could also cause fatigue. See dynamic loads, 5.2.2c.

\section{i) Creep}

- Long term strain, dependent on the load level and thermal environment. This factor can be strongly affected by the other loss and load factors, due to the exponential acceleration of creep with a lineal increase in load level. Creep failures on pristine $12.5 \mathrm{~K}$ Vectran webbing have occurred at 50\% UTS in a year. Even for a robust design that can nominally fail-safe with a primary structural member failure, a rapidly increased TTF may be experienced due to load redistribution into the adjacent webbings or cords. This could lead to a gradually 
accelerating failure propagation. There is no mitigation for creep in a constantly loaded article, other than reducing the operational load on the restraint layer.

5.2.2. Load factors (LdF) - These are factors that increase the effective loads beyond the statically determined pressure loading based on the geometry of the inflatable article.

\section{a) High variance in material strength properties}

- This can lead to components being loaded at a higher percentage of their ultimate load than is designed for when using the tested average breaking strength in the design. This could be mitigated by using the rated minimum or the tested minimum in design but this reduces structural efficiency.

\section{b) Nonuniform load distribution}

- Due to architectural approach and / or level of fabrication fidelity (length setting and build quality that produces the intended geometry to a high degree of accuracy)

- Due to any variations in the post-inflation configuration of the restraint layer after each pressurization. Dependent on architectural approach.

- Due to in-line stiffness variances at rigid structure interfaces, like a hatch or window in the wall of the primary structure that interfaces with multiple webbing or cords

- Mitigation involves precision fabrication and length setting, minimizing friction and interactions between structural softgoods, secure but non-destructive indexing to align layers.

\section{c) Dynamic loading}

- Integrated hard structure adds significant, bulky masses into the lightweight restraint layer shell that can affect the module's dynamic behavior. This could occur on deployment or due to an impact.

- At typical habitat pressures it is unlikely an internal or external event would cause dynamic over-loading of the restraint layer unless an explosion occurred in close proximity to the structure.

- Mitigate by reducing any large cantilevered masses attached to the inflatable.

\section{d) Built-up Inflatable architecture effects}

- Other factors such as friction between elements in the built-up article that effect the loads in the softgoods elements of the restraint layer but don't effect individual component tests. Data for this factor could be explored through sub-scale or multi-component panel-type testing. This is one of the hardest factors to quantify due to the difficulty of accurately measuring these built-up interactive effects between elements. These effects can be minimized by careful design and architecture selection, but there will always be some 
parameters whose effects can only be observed in the full article. Mitigate through building block approach to analysis and test, using results of full-scale testing to adjust analysis.

5.2.3. Additional factors - These are influence factors that may affect the overall design factor but are not classified as a load or loss factor. These are primarily system level risks that must be considered in the formulation of the final design factor but aren't general enough to be rolled into a baseline FOS. These elements are assessed for each design and their combined effect is an Uncertainty Factor (UF) that is an additional multiplier of the design factor.

\section{a) Number and type of samples tested}

- All of the listed loss factors from damage or degradation should be tested with component specimens to ascertain the knockdown factors for strength, and to characterize the impact on the stiffness behavior. A critical factor of that testing is the statistical significance of the results based on their distribution and the number of samples tested. A minimum of 5 samples is prescribed, for example by the ASTM tensile test standard for webbing ${ }^{20}$ for each parameter set test. For tests with high variance a larger number of specimens is preferred.

\section{b) Ability to verify full-scale, as-built restraint layer}

- Measuring load directly in these structures is extremely challenging due to the integration of large and stiff load cells in line with the primary webbings or cords. Strain to load conversion is also difficult due to the load versus strain curve being load-history dependent. There is therefore an uncertainty factor in not being able to verify and validate design loads in the structure by direct measurement.

\section{c) Structural redundancy}

- Redundant load paths, or secondary load paths and rip-stop materials.

- Sectioned off areas of a habitat, or multi-hull approach.

- Redundancy may become a requirement of a human-rated inflatables, like most other human-rated space structures.

\section{d) Ability to repair restraint layer}

- There is a strong likelihood that the restraint layer will not be serviceable due to its placement in the multi-layer shell layup. This significantly increases the required confidence needed in the design factor, to reduce the risk to a level acceptable for a long duration human-rated space structure. Inspection or monitoring via integrated sensors is likely which could be used to reduce the risk of LOC if it can be shown that enough warning could be given to evacuate if necessary prior to a catastrophic failure. It is also possible that the outer layers could be tiled and erected, rather than deployed as they are non-load bearing. This would allow the removal and replacement of damaged MMOD for instance, and allow access to the underlying restraint layer if desired. This could be an approach for 
reducing risk by enabling inspection and possibly repair, although no repair process for a high-strength softgoods structure has currently been demonstrated or proposed.

\section{e) Programmatic controls on fabrication and ground handling}

- To buy down risk and possibly reduce the design factor, a precise, repeatable and cautious fabrication approach is necessary. Programmatic controls to minimize damage and degradation during the build process and packaging for launch is essential. This could be considered a handling factor, where likelihood of human error or mishandling is considered.

\section{f) Experience with design and materials, and level of testing and analysis performed}

- Risk at a system level is proportional to the amount of prior experience, testing and analysis with the architecture and materials being employed. This is a somewhat subjective area on its surface but ties back directly to the load and loss factors, and the overall level of uncertainty in the structure. This factor assumes the structure is not already certified, but that it has significant similarity to a previous design in materials and architecture that allows the narrowing of certain factors based on relevant previous test data and analysis.

- This factor is also relevant to the typical use of commercial off the shelf (COTS) softgoods products in standard sizes. There is some inefficiency in selecting materials this way as they will most likely be oversized for the exact design loads; however it is significantly cheaper, the manufacturer typically has experience with its construction, and it is often based on a government or industry standard versus a custom specification that requires a significant additional investment in testing and acquiring experience through use to reach the same level of confidence as a COTS product.

\section{g) Level of Uncertainty in loss and load factors}

- Depending on the level and depth of testing and analysis performed to characterize the various loss and load factors described above, there is a level of uncertainty in the accuracy of the predictions made that should be accounted for. This acts as a programmatically assigned, catch-all that may or may not be included, depending strongly on the level of confidence in the assessments made on all previous knockdown factors.

The design factor is calculated using the FOS in combination with the loss and load factors and an uncertainty factor based on the combined risks listed under 5.2.3 - additional factors.

Design Factor $=\mathrm{FOS} *\left[\left(\mathrm{LdF}_{1} * \mathrm{LdF}_{2} * \ldots \mathrm{LdF}_{\mathrm{n}}\right) /\left(\mathrm{LsF}_{1} * \mathrm{LsF}_{1} * \ldots \mathrm{LsF}_{\mathrm{n}}\right)\right] * \mathrm{UF}$.

Where: $\quad$ FOS $=$ Factor of Safety $\quad$ UF $=$ Uncertainty Factor

$$
\mathrm{LdF}_{\mathrm{n}}=\text { Load Factors } \quad \mathrm{LsF}_{\mathrm{n}}=\text { Loss Factors }
$$

This design factor multiplied by the limit loads of the softgoods restraint layer elements gives the minimum UTS required of each component to meet the certification. The question remains how 
best to approach obtaining values for these factors, to run the most cost and time efficient tests to produce the most statistically useful data?

\section{Statistical approaches to testing and data analysis}

High-strength softgoods used for human-rated inflatable space structures have high variability in core properties such as strength and load versus strain behavior, which in addition can be effected by the load and loss parameters listed in the previous section. To efficiently and fully test and model the restraint layer elements, an approach is suggested that uses both a systematic method of testing multiple factors at once and a statistically-based analysis to best approximate the as-built article behavior.

Design of experiments (DOE) is a methodology that can be used to plan the most efficient test matrix of specimens given a large set of parameters of interest, and determine via statistical methods what the highest influence factors are. This can allow further testing to concentrate on those factors with the greatest impact on the output parameters of interest, and can expose significant interactions between input factors that would not otherwise appear in single factor tests. In application to testing one of the sets of primary structural components (webbing, cordage, or fabric) the controllable input factors would be the knockdown effects to be studied, such as abrasion, fold damage, and radiation. Uncontrolled factors during the test such as room temperature or humidity should be monitored and ideally these types of factors should be minimized by good test design. The outputs would be load versus strain, and ultimate strength behavior in response to the input factors. For each controllable input factor, the range of input values (levels) to be tested are defined and a test matrix is setup using the factors of interest and their levels. Often only two levels, high and low, are used initially to reduce the number of tests required and to provide a quick assessment of the effects of each factor on the strength behavior (table 1). A two-level, full factorial test consists of 8 runs $\left(2^{3}\right)$ covering each combination of levels and factors. For softgoods, replicate tests at each configuration would be recommended to reduce the effects of variability in determining each factor's influence. Depending on whether this was part of an initial exploratory test set, the number of replicates could be as few as 3 , but 5 is strongly recommended.

This is a simple example, and many excellent books exist on the application of DOE to larger numbers of factors and levels. Fractional factorial tests can also be used to further optimize and reduce the test matrix. These tests produce linear models, but second order or higher response curves or surfaces can also be used to find, in this case, the worst combinations of factors that affect strength (the minimums on the response surface). Using these approaches a useful database of information on the effects of the knockdown factors can be produced and interrogated efficiently from a reasonable number of tests, and values can be assigned to them in a robust manner. These component tests would then feed into sub-component (biaxial panel or sub-scale) and full-scale testing in a building block approach, where analysis, based on the properties found during the component multi-factor testing is used to predict the sub-scale or full-scale behavior, and the results of those tests are then used to update the analysis. 
Accurate predictive modeling and analysis of high-strength inflatable space structures is challenging because of uncertainty and variability in the softgoods properties. Due to these characteristics a strictly deterministic analysis approach isn't generally applicable as the properties are not known to the degree of certainty required. Composite structure certification methods ${ }^{21-23}$ employ statistical methodologies for analysis due to high data scatter in the mechanical properties, and a similar approach is proposed here. Non-deterministic analyses, including stochastic and probabilistic methods use the statistical variability of the material parameters to bound the behavior of the system. For a softgoods module the variability in strength, load distribution, stiffness behavior and frictional properties can be determined via test and input along with their tested probabilistic distribution. This approach, like the multi-factor DOE used for testing can expose sensitivities in the behavior of the article and interactions between properties of elements that might otherwise be missed if, for instance, only minimum or average values were used in a deterministic analysis. A stochastic Finite Element Analysis (FEA) is currently being investigated at NASA LaRC with a model of the Minimalistic Advanced Soft Hatch (MASH) airlock. A fullscale pressure test was performed on this article ${ }^{23}$ with cordage loads and full-field strain recorded for comparison to FEA. Initially, a linear isotropic stochastic model is used to rapidly perform sensitivity studies on the tested bounds of input parameter values. This aids in understanding the characteristics and sensitivities of the system to inputs in the expected range of values. A nonlinear stochastic analysis will then be performed using the bounding nonlinear load versus strain curves for the restraint layer components, which were individually characterized, and the results will be compared to the test results from MASH. Stochastic and probabilistic modeling and analysis is becoming more and more common in tackling complex structures, with high uncertainty or variability due to the ubiquity of powerful computers, and the benefits in understanding, and verifying models of these types of structures.

\section{Conclusions}

This paper is meant as a first step toward a structural certification standard for human-rated inflatable space structures. The focus of this study is on the restraint layer, as it is the primary structural layer of these vessels and the least well characterized in terms of the impact of damage and degradation. In support of that objective, the specific knockdown factors relevant to these structures were identified and categorized. A design factor was then defined by combining a core FOS, based on the criticality of the mission, an architecture specific set of knockdown factors that account for differences in structural approach and mission type, and an uncertainty factor that accounts for system level risks and level of experience. A DOE-based testing approach was presented to efficiently explore and quantify the influence and interactions between the relevant knockdown factors and define their values based on test data. Finally, a stochastic analysis approach was recommended as a way to bound the highly nonlinear and variable material properties used as input, to better understand and model the full-scale article's behavior. These elements could be used in a building block approach, as suggested, to certify the structure of a human-rated inflatable space structure. Over the next year a draft certification standard will be constructed based on this initial study, in coordination with the standards committee at NASA. The goal is to provide NASA and its industry partners with a robust approach to qualifying manned 
space inflatables for flight. Habitable inflatables carry huge potential to fundamentally alter our capabilities to live and work in-space and on planetary surfaces by providing substantially more livable volume than would be otherwise possible.

\section{References}

[1] Von Braun, W., “Crossing the Last Frontier,” Collier’s Magazine, pp. 24-29, 03 -22-52.

[2] Jeppesen, N. L., et al., "Study on Methods of Structurally Evaluating Expandable Structures having Potential Space Applications,” Goodyear Aerospace Corporation, Final Report Contract No. NASw-471, 08-23-63.

[3] Brewer, W. N., and Jeppesen, N. L., "Methods of Evaluation of Inflatable Structures for Space Applications," Goodyear Aerospace Corporation, Contract No. NASw-471, 01-01-64.

[4] Kennedy, K. J., et al., “Inflatable Structures Technology Handbook. Chapter 21: Inflatable Habitats,” JSC-CN$6300,07 / 05 / 00$.

[5] De.la Fuente, H., et al., "TransHab: NASA's Large Scale Inflatable Spacecraft,” 2000 AIAA Space Inflatables Forum; Structures, Structural Dynamics, and Materials Conference, 3-6 April 2000, Atlanta GA, AIAA 20001822.

[6] Kennedy, K. J., et al., “Architecture Baseline for the TransHab Inflatable Module,” Draft JSC Technical Memorandum, 04/05/01.

[7] [ILC Dover paper on bladder work] - Will Add citation here need to look up.

[8] Shortliffe, G., and Christiansen, E., "Mars TransHab Meteoroid and Orbital Debris Shield Performance Assessment,” JSC 27892, June 1997.

[9] Shortliffe, G., and Christiansen, E., "Development Tests of the ISS TransHab Module Meteoroid and Orbital Debris Shield (Phases I to IV),” JSC 28173, June 1997.

[10] Edgecombe, J. E., De la Fuente, H. M., and Valle, G.D., "Damage Tolerance Testing of a NASA TransHab Derivative Woven Inflatable Module,” 2009 AIAA / ASME / ASCE / AHS / ASC Structures, Structural Dynamics, and Materials Conference, AIAA-2009-2167.

[11] Tape and Webbing, Textile, Para-Aramid, Intermediate Modulus Commerical Specification, PIA-T-87130C, 11-25-13.

[12] Airship Design Criteria, FAA-P-8110-2, 02-06-95.

[13] Structural Design and Test Factors of Safety for Spaceflight Hardware, NASA-STD-5001B w/ Change 2, 05$10-16$.

[14] Structural Design Requirements and Factors of Safety for Spaceflight Hardware, JSC 65828 Rev B. Change 1, 07-15-14.

[15] Structural Design and Verification Requirements: International Space Station, SSP-30559 Rev C, 09/29/00.

[16] Fracture Control Requirements for Space Station: International Space Station, SSP-30558 Rev C, 08/24/01.

[17] Bigelow Expandable Activity Module (BEAM) to International Space Station (ISS) Interface Control Document: International Space Station, SSP-57239 Rev A, August 2014.

[18] Dorsey, J. T., “Proposal for Certifying Expandable Planetary Surface Habitation Structures,” NASA-TM2011-217148, May 2011.

[19] Prince, J., et al., “Support for Development of Softgoods Design Factors of Safety,” TI-15-01076, Unpublished NESC Document, 03-25-17.

[20] Standard Test Method for Breaking Strength and Elongation of Textile Webbing, Tape and Braided Material, ASTM-6775-13, 2017. 
[21] Whitehead, R. S., Kan, H. P., and Saether, E. S., "Certification Testing Methodology for Composite Structure Volume I-Data Analysis,” NADC-87042-60, DOT/FAA/CT-86/39, October 1986.

[22] Whitehead, R. S., Kan, H. P., and Saether, E. S., “Certification Testing Methodology for Composite Structure Volume II-Methodology Development,” NADC-87042-60, DOT/FAA/CT-86/39, October 1986.

[23] Composite Materials Handbook - Polymer Matrix Composites, Materials usage, Design and Analysis, Vol 3 of 5, Mil-HDBK-17-3F, 17 June 2002.

[24] Jones, T. C., et al., “Non-Axisymmetric Inflatable Pressure Structure (NAIPS) Full-Scale Pressure Test,” 4th AIAA Spacecraft Structures Conference, AIAA SciTech 2017. January 2017. 